\title{
Implante de bioprótese aórtica "stentless" em pacientes com alterações do anel aórtico
}

Bayard GONTIJO FILHO*, Mário O. VRANDECIC*, Fernando Antônio FANTINI*, Juscelino Teixeira BARBOSA*, João Alfredo de PAULA E SILVA*, Sandra S. AVELAR*, Leonardo F. DRUMOND*, Cristiana GUTIERREZ*

\section{RBCCV 44205-206}

GONTIJO FILHO, B.; VRANDECIC, M. O.; FANTINI, F. A.; BARBOSA, J. T.; PAULA E SILVA, J. A.; AVELAR, S. S.; DRUMOND, L. F.; GUTIERREZ, C. - Implante de bioprótese aórtica "stentless" em pacientes com alterações do anel aórtico. Rev. Bras. Cir. Cardiovasc., 8 (2):118-124, 1993.

RESUMO: Dezessete pacientes portadores de doença da valva aórtica associada a alteraçōes estruturais do anel aórtico foram submetidos a implante de bioprótese aórtica "stentless" (Biocór): 8 pacientes eram portadores de endocardite bacteriana, sendo 3 em valva aórtica e 5 em próteses. Os demais pacientes eram portadores de próteses aórticas disfuncionantes. A técnica de implante foi basicamente a mesma, utilizandose dois niveis de sutura, sendo o primeiro ao nivel do anel aórtico e o outro na parede aórtica. Em 11 pacientes a aorta ascendente foi ampliada com remendo de pericárdio bovino e 3 pacientes foram submetidos, também, a substituição valvar mitral. Houve 1 óbito hospitalar no $23^{\circ}$ dia de pós-operatório por falência de múltiplos órgãos em 1 paciente portador de endocardite bacteriana e quadro de AVC pré-operatório. Dois pacientes necessitaram implante de marcapasso definitivo. Na evolução tardia houve apenas 1 óbito no $6^{\circ}$ mês, de forma súbita, em uma criança portadora de marcapasso. Um paciente desenvolveu deiscência parcial da bioprótese na sutura inferior, o que gerou um gradiente na via de saída do ventrículo esquerdo, sendo reoperado com sucesso de $18^{\circ}$ mês de pós-operatório. Todos os pacientes encontram-se em controle ambulatorial, com estudos ecocardiográficos seriados, que demonstram excelente desempenho da bioprótese "stentless" sem gradientes transvalvares importantes e ausência de regurgitação aórtica significativa.

DESCRITORES: próteses cardíacas artificiais, aórticas, cirurgia.

\section{INTRODUÇÃO}

A substituição da valva aórtica, seja por prótese mecânica ou biológica, apresenta resultados altamente satisfatórios tanto do ponto de vista de morbimortalidade hospitalar, como também de evolução pós-operatória a longo prazo. Apesar disso, complicações são observadas.

As principais complicações encontradas na evolução pós-operatória decorrem de problemas relacionados ao próprio substituto valvar ou a doença valvar aórtica cujas características de exten- são e duração do processo podem determinar um pior prognóstico clínico.

Dentre os problemas relativos à própria doença, encontram-se os pacientes portadores de alterações a nível do anel aórtico, causadas por endocardite bacteriana ou por implantes valvares prévios.

Nessas circunstâncias, o implante dos substitutos convencionais torna-se tecnicamente mais difícil, já que todas essas próteses são fixadas ao nível do anel. Como conseqüência, observa-se maior

Trabalho realizado no Instituto Biocór. Belo Horizonte, MG, Brasil.

Apresentado ao $20^{\circ}$ Congresso Nacional de Cirurgia Cardiaca. Maceió, AL, 2 e 3 de abril, 1993.

- Do Instituto Biocór.

Endereço para separatas: Bayard Gontijo Filho. Caixa Postal 106. 30161-970 Belo Horizonte, MG, Brasil. 
GONTIJO FILHO, B.; VRANDECIC, M. O.; FANTINI, F. A.; BARBOSA, J. T.; PAULA E SILVA, J. A.; AVELAR, S. S.; DRUMOND, L. F.; GUTIERREZ, C. - Implante de bioprótese aórtica "stentless" em pacientes com alterações do anel aórtico. Rev. Bras. Cir. Cardiovasc., 8 (2):118-124, 1993.

incidência de deiscência para-protética (leak) nesse grupo de pacientes. $O$ emprego de homoenxertos ou biopróteses do tipo "stentless" apresenta, nesses casos, uma grande vantagem sobre os outros substitutos, já que sua fixação principal é realizada na parede da aorta e não no anel valvar, diminuindo a possibilidade de leak para-protético.

A bioprótese aórtica "stentless" Biocór, cujas características já foram descritas previamente 5,12 , tem sido empregada no nosso Serviço desde 1990; entre os 88 pacientes, 17 apresentavam alterações estruturais do anel aórtico.

\section{CASUÍSTICA E MÉTODOS}

No período de junho de 1990 a janeiro de 1993 , 88 pacientes portadores de valvopatia aórtica foram submetidos a implante de bioprótese aórtica "stentless" no Hospital Biocór (Belo Horizonte). Nesse grupo, 17 pacientes apresentavam alteração estrutural do anel causada por endocardite em 8, ou por disfunção prévia de bioprótese em 9 pacientes. Os casos de endocardite foram identificados na valva aórtica em 3 pacientes e em prótese aórtica em 5 pacientes. As características destes 17 pacientes estão demonstradas na Tabela 1. Houve predominância do sexo masculino $(94,2 \%)$ e a idade variou de 14 anos a 47 anos (média $=29,4$ ). A grande maioria dos pacientes $(87,5 \%)$ encontrava-se em classe funcional III ou IV da NYHA. A disfunção valvar predominante foi a insuficiência aórtica $(70,5 \%)$, havendo comprometimento severo da função ventricular em $7(43,7 \%)$ pacientes; todos os pacientes foram submetidos a análise ecocardiográfica préoperatória e o cateterismo cardíaco foi realizado em 3 ocasiōes, para avaliação da circulação coronária.

\section{Técnica Cirúrgica}

A abertura do esterno foi realizada com serra

TABELA 1

BIOPRÓTESE "STENTLESS" EM PACIENTES COM ALTERACOÓES DO ANEL AÓRTICO

$$
N=17
$$

oscilante em todos os pacientes, sem complicações. A circulação extracorpórea foi estabelecida com oxigenador de bolhas convencional, sendo empregada hemodiluição parcial, procurando-se manter o hematócrito entre $25 \%-30 \%$. Em todos os pacientes associou-se hipotermia sistêmica $\left(28^{\circ} \mathrm{C}\right)$ e a proteção miocárdica feita com cardioplegia cristalóide (St. Thomas). A bioprótese "stentless" foi implantada através de uma aortotomia transversal, dirigida ao meio do seio de Valsalva não coronariano. Nos pacientes com endocardite, os abscessos e o tecido necrótico foram extensivamente desbridados e lavados com solução anti-séptica. A fixação inferior da "stentless" foi realizada com pontos separados na área correspondente ao sistema de condução e com chuleio no restante. Nos casos de descontinuidade aorto-ventricular causado por processo infeccioso, a fixação foi feita na parte superior do septo interventricular e na base da cúspide anterior da valva mitral, em área segura para se ancorar os pontos.

Em seguida, procedeu-se à fixação superior da "stentless" na parede aórtica, com chuleio, contornando-se com cuidado os óstios coronários. Desta forma, os pacientes portadores de deformidades importantes do anel aórtico tinham a raiz da aorta reconstituída pelo próprio substituto (Figuras 1 e 2). Em 11 pacientes a aorta ascendente foi ampliada com enxerto de pericárdio bovino, utilizado para fixação da prótese na sua porção correspondente ao seio de Valsalva não coronariano (Figuras 3,4 e 5). Em um paciente foi associada a troca de valva mitral e, em outros dois, retroca mitral por degeneração da bioprótese.

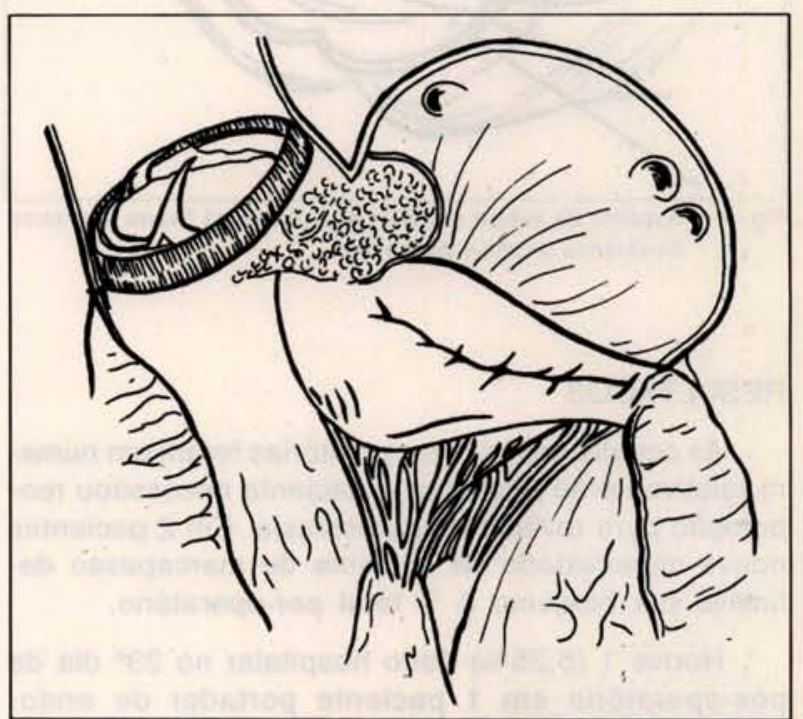

Fig. 1 - Endocardite em prótese aórtica com deiscência e abscesso anular. 
GONTIJO FILHO, B.; VRANDECIC, M. O.; FANTINI, F. A.; BARBOSA, J. T.; PAULA E SILVA, J. A.; AVELAR, S. S.; DRUMOND, L. F.; GUTIERREZ, C. - Implante de bioprótese aórtica "stentless" em pacientes com alterações do anel aórtico. Rev. Bras. Cir. Cardiovasc., 8 (2):118-124, 1993.

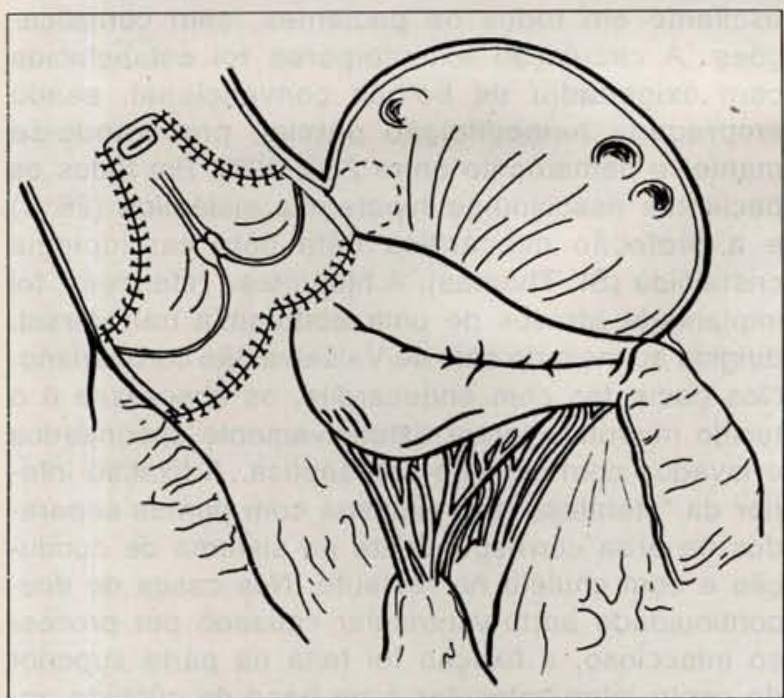

Fig. 2. Tratamento do abscesso anular com reconstrução da raiz aórtica com bioprótese "stentless".

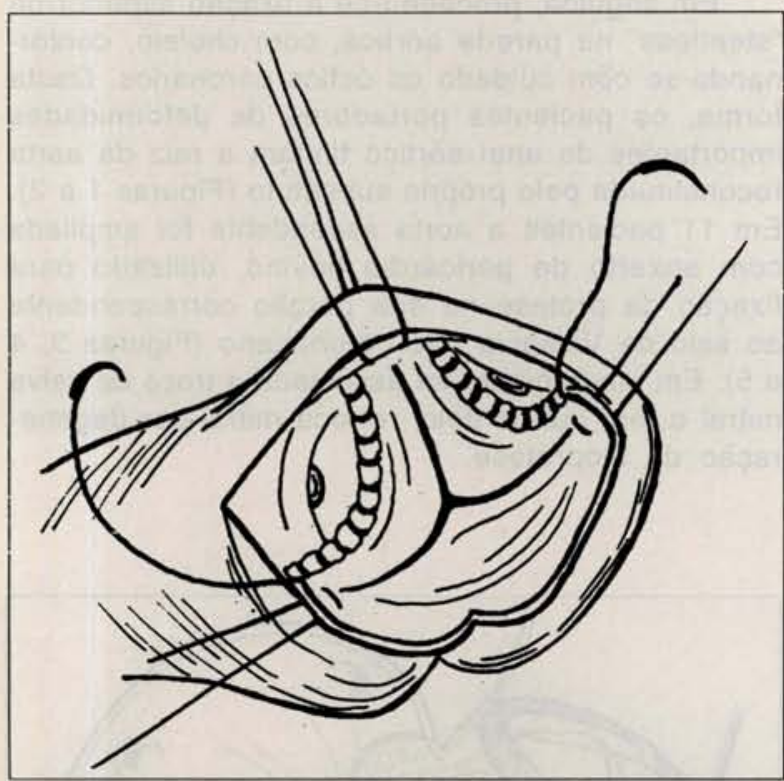

Fig. 3 - Aspecto da sutura superior da prótese, já fixada aos seios coronários direito e esquerdo.

\section{RESULTADOS}

As complicações pós-operatórias foram em número relativamente pequeno; 1 paciente necessitou reoperação para revisão de hemostasia. Em 2 pacientes houve necessidade de implante de marcapasso definitivo por bloqueio $\mathrm{A} V$ total per-operatório.

Houve $1(6,25 \%)$ óbito hospitalar no $23^{\circ}$ dia de pós-operatório em 1 paciente portador de endocardite grave, com quadro de AVC isquêmico ocorrido antes da operação, o qual se agravou no pós- operatório, evoluindo para falência de múltiplos órgãos. Houve 1 óbito no $6^{\circ}$ mês de pós-operatório em paciente portador de marcapasso, de forma súbita. Os demais pacientes encontram-se em controle ambulatorial; houve melhora significativa da classe funcional em todo o grupo, e apenas 3 pacientes encontram-se em classe funcional II.

\section{Avaliação Ecocardiográfica Pós-Operatória}

Todos os pacientes foram submetidos a estudos ecocardiográficos periódicos no pós-operatório. Com relação à função da prótese, a velocidade máxima (Vmax) variou de $1.2 \mathrm{~m} / \mathrm{s}$ a $3.0 \mathrm{~m} / \mathrm{s}$ com uma

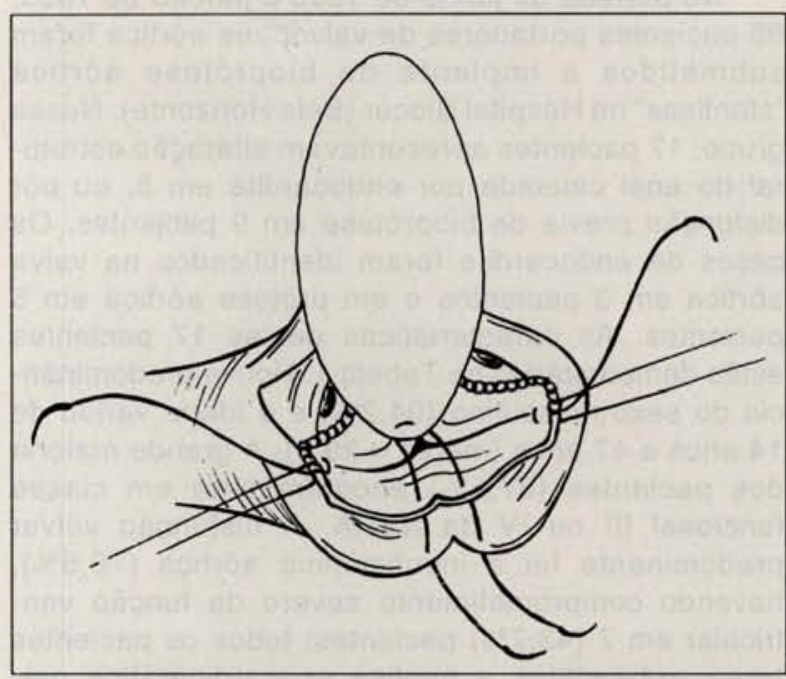

Fig. 4 - Ampliação da aorta ascendente com enxerto de pericárdio e concomitante fixaçào da protese (seıo náo-coronarıo)

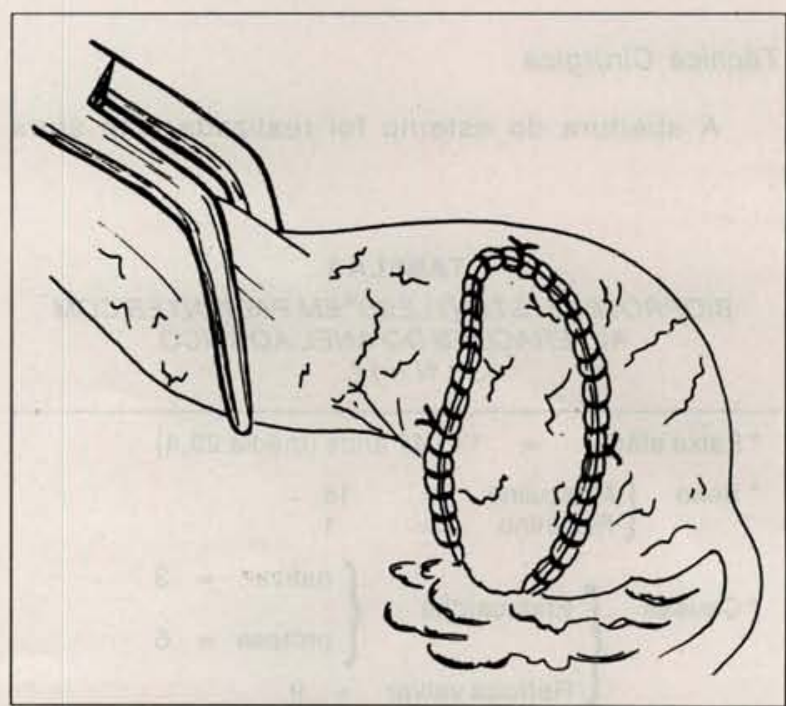

Fig. 5 - Aspecto final da ampliação da aorta ascendente. 
GONTIJO FILHO, B.; VRANDECIC, M. O.; FANTINI, F. A.; BARBOSA, J. T.; PAULA E SILVA, J. A.; AVELAR, S. S.; DRUMOND, L. F.; GUTIERREZ, C. - Implante de bioprótese aórtica "stentless" em pacientes com alteraçōes do anel aórtico. Rev. Bras. Cir. Cardiovasc., 8 (2):118-124, 1993.

média de $2.17 \mathrm{~m} / \mathrm{s}$. O gradiente médio transvalvar variou de $3.4 \mathrm{mmHg}$ a $22.0 \mathrm{mmHg}$, com média de $10,6 \mathrm{mmHg}$. (Tabela 2). Nenhum paciente apresentou insuficiência aórtica significativa, observando-se apenas refluxo mínimo em 2 pacientes e leve em outros dois. Nos exames seriados, todos os pacientes demonstraram desempenho estável da prótese com manutenção dos mesmos dados ecocardiográficos. Apenas o paciente que foi reoperado com deiscência parcial da sutura inferior evidenciou um gradiente crescente na via de saída do $V E$, que foi corrigido após a segunda intervenção.

A análise ecográfica dos pacientes portadores de abscessos e descontinuidade aorto-ventricular demonstrou excelente reconstrução da raiz aórtica com a "stentless" (Figuras 6 e 7).

\section{COMENTÁRIOS}

A incidência de deiscência para-protética (leak) após substituição da valva aórtica pode atingir $17 \%$

TABELA 2

BIOPRÓTESE AÓRTICA "STENTLESS" EM PACIENTES COM ALTERAÇÕES DO ANEL AÓRTICO

\begin{tabular}{ll}
\hline \multicolumn{2}{c}{ Dados ecocardiográficos pós-operatórios } \\
\hline *Gradiente transvalvar & $=3,4-22,0 \mathrm{mmHg}$ \\
& $($ média $=10,6 \mathrm{mmH})$ \\
*Velocidade máxima & $=1,2-3,0 \mathrm{~m} / \mathrm{s}$ \\
& (média $=2,17 \mathrm{~m} / \mathrm{s})$ \\
$*$ Insuficiência aórtica & $\left\{\begin{array}{l}\text { Ausente }=13 \\
\text { Mínima }=2 \\
\text { Leve }=2\end{array}\right.$ \\
\hline
\end{tabular}

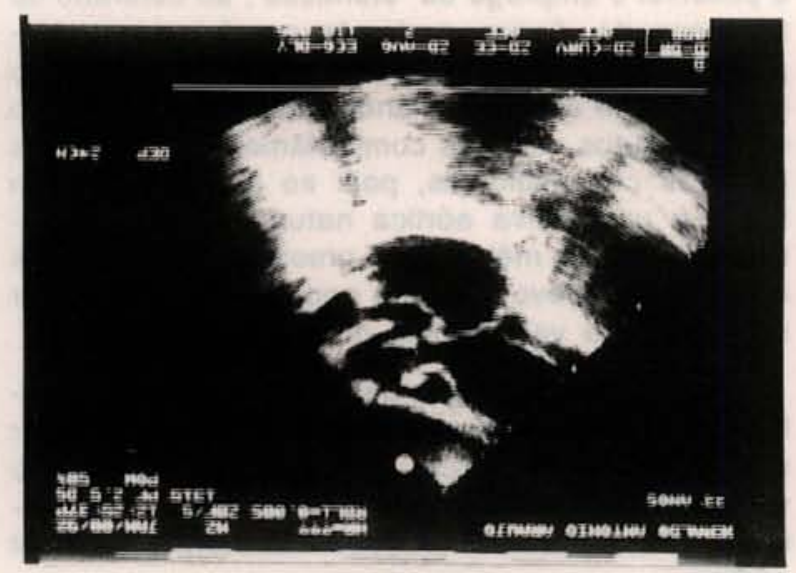

Fig. 6 - Aspecto ecocardiogrático de paciente com endocardite em prótese e acometimento extenso da raiz aórtica. dos casos ${ }^{7}$. Tal complicação pode ser de pequena significação hemodinâmica, ou pode levar a grave insuficiência aórtica, às vezes de instalação rapidamente progressiva, principalmente na presença de processo infeccioso.

Em casos extremos, pode haver deiscência total da prótese, com embolização distal da mesma. As circunstâncias principais que propiciam o aparecimento de leak para-valvar, são as alteraçōes estruturais do anel aórtico, cujas causas principais são a endocardite bacteriana e a substituição valvar prévia. Enquanto a cada troca valvar temos maior chance de deiscência pelo desgaste progressivo do anel aórtico, as endocardites aumentam em cinco vezes essa possibilidade 7 .

A endocardite da valva aórtica pode atingir o anel aórtico em maior ou menor extensão, dependendo basicamente da duração do processo e do agente bacteriano. Já na endocardite em prótese aórtica, o acometimento do anel é bem mais extenso e o agente etiopatogênico é, com freqüência, o Staphilococcus aureus, o que torna o processo ainda mais grave. As grandes destruições do anel aórtico, embora bem menos freqüentes atualmente, devido a intervenção mais precoce, constituem um verdadeiro desafio ao cirurgião. A presença de abscessos, de comunicações intercavitárias e a descontinuidade aorto-ventricular causada pelo processo infeccioso são situações que exigem perícia e criatividade do cirurgião para abordá-las.

Técnicas cirúrgicas mais radicais podem ser necessárias, tais como a reconstrução da via de saída do ventrículo esquerdo com conduto valvado 10 , a translocação da valva aórtica com revascularização miocárdica ${ }^{2}$, ou as reconstituições extensas envolvendo o anel mitral e aórtico ${ }^{4}$. Evidente-

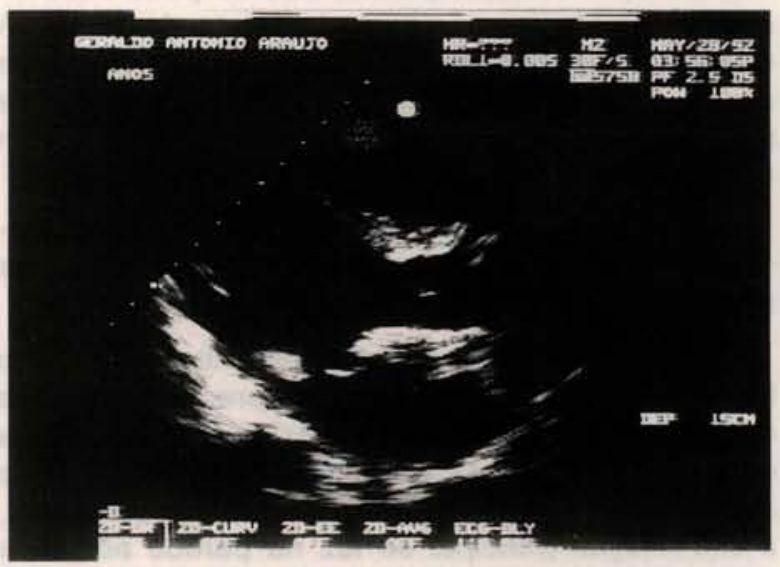

Fig. 7 - Aspecto ecocardiográfico pós-operatório do mesmo paciente evidenciando uma reconstrução completa da raiz aórtica. 
GONTIJO FILHO, B.; VRANDECIC, M. O.; FANTINI, F. A.; BARBOSA, J. T.; PAULA E SILVA, J. A.; AVELAR, S. S.; DRUMOND, L. F.; GUTIERREZ, C. - Implante de bioprótese aórtica "stentless" em pacientes com alterações do anel aórtico. Rev. Bras. Cir. Cardiovasc., $8(2): 118-124,1993$.

mente, todos esses procedimentos apresentam maior índice de morbimortalidade per e pós-operatória. 0 uso dos substitutos valvares convencionais, nessas situações, apresenta dois grandes inconvenientes; em primeiro lugar a fixação torna-se muito mais difícil, já que todos os modelos têm sua implantação realizada ao nível do anel aórtico. O outro inconveniente é a presença de material inerte como o tecido de revestimento, o que parece aumentar o índice de infecção no pós-operatório ${ }^{6}$.

Vários trabalhos demonstram a superioridade do homoenxerto aórtico sobre as próteses convencionais, tanto nos casos de endocardite, como nas reoperações para retrocas valvares $6-9,11,13$.

Além de ser definitivamente mais resistente à infecção, por ser constituído apenas de tecido biológico, o uso do homoenxerto está associado a menor incidência de leak para-valvar, pois a sua fixação principal se faz na parede aórtica ${ }^{7}$. Os homoenxertos também têm uma fixação ao nível do anel aórtico, porém, ao contrário das próteses comuns, esta sutura não está submetida a nenhuma tensão hidrodinâmica. O homoenxerto é, também, bastante versátil nas reconstruções mais extensas da aorta ascendente. A cúspide anterior da valva mitral pode ser usada para fechamento da comunicação interventricular; já a manutenção da parede aórtica acima do plano valvar possibilita a sua utilização como conduto valvado.

As desvantagens principais dos homoenxertos são as dificuldades na sua aquisição, a técnica de implante mais trabalhosa, exigindo maior treinamento do cirurgião, e a degeneração a longo prazo.

A ocorrência de insuficiência aórtica $(25 \%$ em 10 anos) é um dos principais problemas do homoenxerto no pós-operatório tardio 9 .

A causa principal da insuficiência aórtica é a degeneração tissular, porém, não se pode subestimar os problemas técnicos durante o implante. A distorçāo do homoenxerto durante a fixação, bem como a utilização em aneis aórticos muito dilatados pode causar insuficiência aórtica importante, com necessidade de reintervenção.

Essas dificuldades observadas com os homoenxertos têm proporcionado o desenvolvimento de novos substitutos aórticos 1,3 .

A bioprótese aórtica "stentless" Biocór, em uso há 30 meses no nosso Serviço, apresenta algumas características dos homoenxertos, que a tornam uma excelente opção para pacientes com ateraçōes do anel aórtico. Da mesma forma que os homoenxertos, a sua fixação principal se faz na parede aórtica e não no anel valvar; além disso, não apresenta te- cido inerte na sua confecção, sendo totalmente composta de tecido biológico, já que as válvulas são sustentadas por um tubo de pericárdio bovino. Essas vantagens podem ser atestadas na nossa série, pela ausência de deiscência para-valvar, como também pela inexistência de recorrência de endocardite. Houve apenas 1 paciente portador de endocardite nativa, que apresentou deiscência parcial da sutura inferior, identificada pelos estudos ecocardiográficos seriados no pós-operatório. Este fato ocorreu no início da experiência, quando empregávamos sutura contínua na fixação da parte inferior da prótese, substituída potencialmente para pontos separados, não se verificando mais o problema. O paciente, apesar de assintomático, foi reoperado devido a presença, de gradiente importante na via de saida do ventrículo esquerdo, causado pela protusão do tecido valvar durante a sístole. A fixação da prótese, nesse pequeno segmento, corrigiu totalmente 0 problema.

Ressalte-se o perfeito estado em que se encontravam as válvulas durante a reoperação.

A ausência de insuficiência aórtica significativa deve ser destacada no seguimento dos pacientes submetidos ao implante da "stentless". Em todo o grupo de pacientes operados até janeiro de 1993 $(n=88)$, apenas 1 paciente apresentou insuficiência aórtica leve e moderada, sem repercussão hemodinâmica. Os demais, inclusive aqueles do presente relato, apresentam excelente desempenho da bioprótese, sem nenhum sinal de insuficiência aórtica progressiva. Essa diferença com relação aos homoenxertos é explicada pela característica da confecção da bioprótese "stentless"; são selecionadas três válvulas porcinas isoladas, discretamente redundantes com relação ao tamanho do anel, o que evita que qualquer distorção da orientação valvar durante o implante interfira na coaptação. Desta forma, mesmo em pacientes com grandes anéis aórticos, é possível o emprego da "stentless", ao contrário do homoenxerto. Assim, podemos concluir que a bioprótese "stentless" aórtica se aproxima em muito do homoenxerto aórtico, estando ambos em um grupo de substitutos valvares completamente diverso das próteses convencionais, pois se assemelham em muito a uma valva aórtica natural. O desenvolvimento de novos métodos de preservação de tecidos aliado a este novo desenho poderá, ainda, oferecer um substituto valvar mais duradouro.

A experiência adquirida nesses meses de utilização da bioprótese aórtica "stentless" nos faz crer que se trata de uma boa alternativa na substituição valvar aórtica, particularmente no grupo de pacientes portadores de alteraçōes estruturais do anel aórtico. 
GONTIJO FILHO, B.; VRANDECIC, M. O.; FANTINI, F. A.; BARBOSA, J. T.; PAULA E SILVA, J. A.; AVELAR, S. S.; DRUMOND, L. F.; GUTIERREZ, C. - Implante de bioprótese aórtica "stentless" em pacientes com alteraçōes do anel aórtico. Rev. Bras. Cir. Cardiovasc., 8 (2):118-124, 1993.

\section{RBCCV 44205-206}

GONTIJO FILHO, B.; VRANDECIC, M. O.; FANTINI, F. A.; BARBOSA, J. T.; PAULA E SILVA, J. A.; AVELAR, S. S.; DRUMOND, L. F.; GUTIERREZ, C. - Aortic valve replacement with a stentless bioprosthesis in patients with weakened aortic annulus. Rev. Bras. Cir. Cardiovasc., 8(2):118-124, 1993.

ABSTRACT: An aortic stentless bioprosthesis (Biocor Ind.) was implanted in 17 patients with difficult aortic annulus due either to endocarditis or to a previous aortic valve replacement. Native valve endocarditis was present in 3 patients and prosthetic valve endocarditis in $5 ; 9$ patients had one or more previous aortic valve replacements showing a wheakened aortic rim. The stentless bioprosthesis was implanted with a two layers suture technique similar to a homograft implant. The ascending aorta was enlarged with a patch of bovine pericardium in 11 ocasions; mitral valve replacement was concomitantly performed in 3 patients. There was one hospital death secondary to multiple organ failure in a patient with acute endocarditis and a preoperative cerebro-vascular accident. One patient who was pacemaker dependant had a sudden death in the 6 th postoperative month. All other 15 patients are in regular follow-up with periodic ecocardiographic analysis. One patient had partial deiscense of the lower suture line which generated a gradient in the left ventricular outflow tract; he was reoperated upon and the valve was refixed with flow normalization. In all patients the valve has shown an excellent performance with very small gradients (mean $=10.6 \mathrm{mmHg}$ ) and abscence of significant aortic regurgitation. No case of reinfection was observed in the patients with endocarditis. The stentless aortic bioprosthesis proved to be an excellent heart valve substitute in this difficult group of patients.

DESCRIPTORS: heart valves prosthesis, aortic, surgery.

\section{REFERÊNCIAS BIBLIOGRÁFICAS}

BATISTA, R. J. V.; DOBRIANSKIJ, A.; COMAZZI Jr., M.; LESSA NETO, L. T.; ROCHA, G.; SARTORI, F.; WESTPHAL, R.; TIMI, J. R.; MOREIRA, R.; OLIVEIRA, P. F. - Clinical experience with stentless pericardial aortic monopatch for aortic valve replacement. J. Thorac. Cardiovasc. Surg., 93: 1926, 1987.

2 DANIELSON, G. K.; TITUS, J. L.; DUSHANE, J. W. Successful treatment of aortic valve endocarditis and aortic root abscesses by insertion of prosthetic valve in ascending aorta and placement of bypass grafts to coronary arteries. J. Thorac. Cardiovasc. Surg., 67: 443-449, 1974.

3 DAVID, T. E.; POLLICK, C.; BOS, J. - Aortic valve replacement with stentless porcine aortic bioprosthesis. J. Thorac. Cardiovasc. Surg., 99: 113-118, 1990.

4 ERGIN, M. A.; RAISSI, S.; FOLLIS, F.; LANSWAN, S. L.; GRIEPP, R. B. - Annular destruction in acute bacterial endocarditis: surgical techniques to meet the challenge. J. Thorac. Cardiovasc. Surg., 97: 755-763, 1989.

GONTIJO FILHO, B.; VRANDECIC, M.; MOREA, M.; RADEGRAN, K.; PAULA E SILVA, J. A.; FANTINI, F. A.; BARBOSA, J. B. - Nova bioprótese aórtica em suporte: resultados clínicos. Rev. Bras. Cir. Cardiovasc., 7: 208-214, 1982.
6 HAYDOCK, D.; BARRATT-BOYES, B. G.; MACEDO, T.; KIRKLIN, J. W.; BLACKS, E. - Aortic valve replacement for active infectious endocarditis in 108 patients: a comparison of freehand allograft valves with mechanical prostheses and bioprostheses. J. Thorac. Cardiovasc. Surg., 103: 130-139, 1992.

7 KIRKLIN, J. W. \& BARRAT-BOYES, B. G. - Aortic valve disease. In: KIRKLIN, J. W. \& BARRAT-BOYES, B. G. (eds.) Cardiac surgery. New York, Wiley, 1986. p. 373-429.

8 KIRKLIN, J. W. \& PACIFICO, A. D. - Aortic valve endocarditis with aortic root abscess cavity: surgical treatment with aortic valve homograft. Ann. Thorac. Surg., 45: 674-679, 1988.

9 MATSUKI, O.; ROBLES, A.; GIBBS, S.; BODNAR, E.; ROSS, D. N. - Long term performance of 555 aortic homografts in aortic position. Ann. Thorac. Surg., 46: 187-191, 1988.

10 RANDOLPH, J. D.; TOAL, K.; STELZER, P.; ELKINS, R. C. - Aortic valve and left ventricular outflow tract replacement using allograft and autograft valves: a preliminary report. Ann. Thorac. Surg., 48: $345-$ $349,1989$.

11 TIENA, I. C.; ORSZULAK, J. A.; SCHAFF, H. V.; DANIELSON, G. K. - Results of homograft aortic valve replacement for active endocarditis. Ann. Thorac. Surg., 49: 619-624, 1990. 
GONTIJO FILHO, B.; VRANDECIC, M. O.; FANTINI, F. A.; BARBOSA, J. T.; PAULA E SILVA, J. A.; AVELAR, S. S.; DRUMOND, L. F.; GUTIERREZ, C. - Implante de bioprótese aórtica "stentless" em pacientes com alteraçōes do anel aórtico. Rev. Bras. Cir. Cardiovasc., 8 (2):118-124, 1993.

12 VRANDECIC, M.; MOREA, M.; RADEGRAN, K.; GONTIJO FILHO, B.; PAULA E SILVA, J. A.; FANTINI, F. A.; BARBOSA, J. T. - Clinical use of a new stentless porcine aortic bioprosthesis: a multicenter study. In: Cardiology and cardiac surgery: current topics. New York. Futura Publishing Co. Inc,
1992. p. 23-234.

13 ZWISCHENBERG, J. B.; SHALABY, T. Z.; CONTI, V. R. - Viable cryopreserved aortic homograft for aortic valve endocarditis and annular abscesses. Ann. Thoracic. Surg., 48: 365-370, 1989. 